\title{
A study on the outcome of pulse therapy in vesicobullous disorders at a tertiary care centre in Chidambaram, Tamilnadu
}

\author{
Kannambal K., ${ }^{, *}$, Prasad P. V. S. ${ }^{2}$, Kaviarasan P. K. ${ }^{3}$, Poorana B. ${ }^{4}$, Ranjani D. ${ }^{5}$ \\ ${ }^{1}$ Associate Professor, ${ }^{2}$ Professor, ${ }^{3}$ Professor \& HOD, ${ }^{4}$ Assistant Professor, ${ }^{\mathbf{5}}$ Junior Resident, Dept. of Dermatology, Rajah \\ Muthiah Medical College, Annamalai University, Tamilnadu, India
}

Corresponding Author:

Email: sharm_kr@yahoo.com

\begin{abstract}
Introduction: With the advent of pulse therapy and adjuvant immunosuppressive drugs, the outcome and prognosis of vesicobullous disorders have been improved to a greater extent. Different regimen protocol has been tried and this descriptive study was carried out to assess the outcome of various pulse therapy regimens in vesicobullous disorder at our centre.

Methods: A total of 26 vesicobullous patients were enrolled for the study. Diagnosis was made clinically and confirmed by histopathology. They received treatment with Dexamethasone-Cyclophosphamide pulse (DCP) or Dexamethasone -Azathioprine pulse (DAP) or Dexamethasone pulse (DP) therapy and were followed up for clinical remission and side effects of therapy.

Results: There was a total of 26 patients comprising $69.23 \%$ females and $30.77 \%$ males and $50 \%$ were between the age group of 40-50 years. Pemphigus vulgaris was the commonest clinical type seen in 69.23\%.DCP was given for 22(84.62\%) patients, dexamethasone pulse in 3(11.54\%) and DAP in one $(3.85 \%)$. In DCP group $77.27 \%$ of patients were in Phase I and $22.73 \%$ achieved clinical remission. One patient was refractory to DCP and required rituximab infusion. Weakness, headache, vomiting and weight gain were the commonest side effects encountered in our study. Avascular necrosis of femur and reactivation of pulmonary tuberculosis were the major side effects noticed in one patient each.

Conclusion: DCP therapy prove to be an excellent therapeutic regimen in case of vesicobullous disorders to attain faster recovery sparing the adverse effect of corticosteroids. DAP can be an useful therapeutic option in patients who were unmarried or in reproductive age group.
\end{abstract}

Keywords: Pemphigus vulgaris, Dexamethasone-cyclophosphamide pulse. Dexamethasone-azathioprine pulse, Dexamethasone pulse.

\section{Introduction}

Autoimmune blistering disorders are potentially life-threatening disorders with significant mortality. Dexamethasone-cyclophosphamide (DCP) designed by Pasricha et al, has become the standard treatment protocol since its introduction. ${ }^{1}$ Addition of immunosuppressive agents like cyclophosphamide, azathioprine, mycophenolate mofetil has further reduced the mortality to less than $5 \% .^{2}$ As the treatment needs to be patient-specific, different regimens and modifications have been tried in order to attain faster remission with least side effects. In this article, we discuss the observations and the results of various pulse therapies in vesicobullous disorders.

\section{Materials and Methods}

A descriptive study was conducted during the period of August 2014 to August 2017. Ethical clearance was sought from institutional ethical committee. After acquiring informed consent, about 26 vesicobullous patients who attended the dermatology outpatient department of Rajah Muthiah Medical College, Chidambaram were selected for the study, irrespective of the severity of the disease. Pregnant, lactating women and patients with uncontrolled diabetes, hypertension, cardiac, liver and renal disease were excluded from the study. Detailed history and complete dermatological examination were done in all cases. Tzanck smear and skin biopsy for histopathological examination were done to confirm the diagnosis. Pre-pulse evaluation was done with necessary investigations like complete hemogram, urine routine, liver function test, renal function test, blood sugar, electrocardiogram, chest X-ray, Mantoux test, enzyme linked immunosorbent assay (ELISA) for HIV were done in all cases to rule out systemic illness, comorbidities and infections.

In this study, patients were instituted with either Dexamethasone-Cyclophosphamide pulse (DCP) or Dexamethasone -Azathioprine pulse (DAP) or Dexamethasone pulse (DP) therapy based on their severity of skin and mucosal lesions and marital status. ${ }^{1}$

1. Dexamethasone-cyclophosphamide pulse (DCP): The DCP constitutes $100 \mathrm{mg}$ of dexamethasone in $500 \mathrm{ml}$ of $5 \%$ dextrose given as a slow intravenous infusion over two hours for three consecutive days along with cyclophosphamide $500 \mathrm{mg}$ infusion on one of the days. Between the pulse patients were given 50mg of cyclophosphamide orally every day. DCP regimen is broadly divided into 4 phases. Phase I lasts until the patient goes into clinical remission and without intervening corticosteroids. In Phase II, patient remains completely normal but receives DCP along with daily oral cyclophosphamide $50 \mathrm{mg}$ for nine months. In Phase III, only oral cyclophosphamide $50 \mathrm{mg}$ will be 
continued for another 9 months followed by Phase IV, which should be treatment free follow up period as long as possible.

2. Dexamethasone-azathioprine pulse (DAP): In this, cyclophosphamide was replaced by $50 \mathrm{mg}$ of azathioprine daily during the first three phases. No bolus dose of azathioprine was given during the pulse. DAP was initiated in those who were unmarried and had not completed their family.

3. Dexamethasone only pulse (DP): Only 100mg of dexamethasone in 5\% dextrose as slow intravenous infusion were given for three consecutive days. DP was instituted in patients with less severe disease.

Minor modifications in DCP were:

a. All patients with $>50 \%$ BSA and mucosal involvement were treated for intercurrent infections with systemic antibiotics and antifungals for skin and mucosa respectively. Raw areas and erosions were addressed with topical antibiotics, soaks and compresses. Patients were also advised to maintain their oral hygiene with regular brushing and antiseptic mouth washes every time after they had meal and skin hygiene with gentle cleansers/shampoos.

b. Supportive drugs were given to all patients with oral calcium $500 \mathrm{mg}$ daily along with Vitamin D3 at a dose of 60,000 IU once per week during three phases.

All diabetic patients who were under adequate control were given $8 \mathrm{U}$ of crystalline insulin via an intravenous drip during the pulse. If the disease onset is very severe or development of new lesions $>5 /$ day with regular pulse, either interpulse with dexamethasone $100 \mathrm{mg}$ intravenously or daily prednisolone at a starting dose of $1-2 \mathrm{mg} / \mathrm{kg} /$ day which was slowly tapered to $50 \%$ of the initial dose every two weeks when $80 \%$ lesions showed re-epithelization. Mycophenolate mofetil at a dose of $1 \mathrm{~g} /$ day was added if there is poor response even with DCP/DAP.

The evaluation of response to treatment included both clinical and laboratory analysis. Clinical remission was defined as having no lesions on the skin or mucosa. Any deviation from 28 days cycle was considered as an irregular treatment. Patients on irregular treatment were given adequate counseling and advised regular follow up.

\section{Results}

A total of twenty six vesicobullous patients were studied from Aug 2014 to Aug 2017. Patients were aged from 25-75 years. The age and sex wise distribution are shown in tables $1 \& 2$ (Table $1 \& 2$ ) respectively. Eighteen patients (69.23\%) had pemphigus vulgaris, followed by pemphigus foliaceus in five $(19.23 \%)$ and bullous pemphigoid in 3 patients $(11.54 \%)$. The body surface area involvement is shown in table 3 (Table 3).
DCP was given for twenty-two(84.62\%) patients, dexamethasone pulse in three $(11.54 \%)$ and DAP in one $(3.85 \%)$ patient who had primary infertility. The various types of pulse regimen administered were shown in table 4 (Table 4 ).

In the DCP subgroup with severe lesions, four patients $(18.18 \%)$ received interpulse with DP for two days and three patients $(13.64 \%)$ required oral steroids in addition ranging from 10-60mg/day during Phase- 1 . One patient who was refractory to DCP, was given intravenous rituximab as per Rheumatoid arthritis protocol which was subsequently followed with regular dexamethasone pulse alone. Three patients $(13.64 \%)$ in DCP group developed pulmonary tuberculosis, macular choroiditis and avascular necrosis of femur respectively and were discontinued from the study.

The minor and major side effects observed with various pulse regimens are shown in table 5 and table 6 (Table 5 and 6) respectively. The most common observed being weakness in sixteen, gastrointestinal upset in nine and weight gain in ten patients respectively. Raised blood sugar levels and electrolyte abnormalities were noticed in eight and seven patients respectively on days 2,3 and 4 which returned to normal values after completion of the pulse. Amenorrhoea was observed in 8 out of 18 females, probably induced by cyclophosphamide.

\section{Discussion}

Pemphigus is considered as a leading cause of acute skin failure because of its consequences. Pulse therapy has made a revolution in the treatment of pemphigus. $^{3}$ In our study $69.23 \%$ of patients had pemphigus vulgaris, $19.23 \%$ had pemphigus foliaceus and $11.54 \%$ had bullous pemphigoid. This percentage is similar to those reported by Micaliet al. ${ }^{4}$

In our study, among the pemphigus group,eight were younger than 40 years of age and four were older than 60 years, depicting that pemphigus vulgaris is seen over a wider age span, which is also consistent with other study. 4

There was a pronounced female predominance with female: male ratio of 2.3:1 suggesting the role of hormonal factors like estrogen which can predispose autoimmunity as proposed by Coombs and Taylorin their study. ${ }^{5,6}$

Pulse therapy has replaced the dependence of conventional steroids for the management of autoimmune vesicobullous disorders. The advantages include rapid healing, reduced morbidity, short hospital stay and absence of common side effects of daily corticosteroid therapy. ${ }^{7,8}$ In our study, out of twenty two patients who received DCP, five $(22.73 \%)$ patients did not have any relapse after entering Phase II. They completed Phase I in less than 18 months. Seventeen $(77.27 \%)$ patients who received DCP were still in Phase I, the probable reason for this may be due to poor 
compliance as many were from rural areas with difficulty to access and hence irregular follow up. ${ }^{9}$

Four patients $(18.18 \%)$ in the study group required interpulse with dexamethasone during Phase I for three days after two weeks of regular DCP as they were improving slowly to the routine DCP. Three patients required daily steroids inspite of DCP. These patients had severe mucosal involvement. This was in accordance with a previous critically evaluated study showing $60 \%$ of patients with high disease activity and increased antibody titres may require extra doses of steroids to achieve clinical remission. ${ }^{10}$ However use of very high, intermediate and low dose corticosteroids have their own advocates. ${ }^{11}$ One female patient had persistent skin and mucosal lesions even after following all the steroid regimens and immunosuppressives and required rituximab infusion. She was in complete remission with rituximab and is maintained only on DP.

DP \& DAP proved to be beneficial especially in younger age to achieve continued remission. ${ }^{9}$ This regimen prevents from the gonadal toxicity of cyclophosphamide which manifests as amenorrhoea, azoospermia and infertility within six months of therapy. ${ }^{12}$ Eight out of eighteen females (44.44\%) in our study had amenorrhoea following DCP, which is in contrast with an earlier study where they have reported in about $85 \% .^{13}$ Hemorrhagic cystitis and bladder carcinoma were not noticed in our study as they are known to occur only after a cumulative dose of $85 \mathrm{~g}$ of cyclophosphamide. ${ }^{14}$

The reported serious side effects of pulse therapy include arrythmias, seizures, and sudden death. The serious complication encountered during our study was avascular necrosis of femur neck in one. This can be prevented by regular monitoring of bone mineral density and addition of bisphosphonates and calcium supplements, ${ }^{15}$ but symptoms and radiographic features resolved after six months of rest and supportive therapy.

Other adverse reactions observed were hypertension (15.38\%), hyperglycemia $(30.77 \%)$ and electrolyte imbalance (26.92\%). No new case of diabetes was diagnosed in previously normoglycemic patients on DCP regimen similar to a study done by
Zivanovicet al. ${ }^{13}$ Slow infusion over 4-5 hours has minimized many serious side effects. Regular monitoring of vitals and blood sugar levels may help on early detection and treatment.

In our study, we noted minor side effects like headache $(46.15 \%)$, vomiting $(34.62 \%)$, weight gain (38.46\%), weakness $(61.54 \%)$ with a higher prevalence than that of a previous case series as observed by Kandan et al. ${ }^{16}$ However, these problems required supportive treatment along with rest and did not recur subsequently.

Intercurrent infections were seen in $11.54 \%$. The commonest was secondary pyogenic infection and oral candidiasis which were in accordance with study by Kandan et al. ${ }^{16}$ Pus culture revealed Methicillin Resistant Staphylococcus aureus (MRSA) and hyphae in potassium hydroxide $(\mathrm{KOH})$ mount. These were common in patients with severe mucosal involvement. Prophylactic antibiotics and antifungals may play a pivotal role in such patients and were supplemented to these patients. ${ }^{17}$ Pulmonary TB was reactivated in one patient which warranted anti-tuberculous therapy and discontinuation of pulse therapy. ${ }^{18}$

There was no mortality in our study, either due to disease or therapy related. Mahajanet al also did not report any mortality. ${ }^{9}$

The oral mucosal lesions in general were more recalcitrant to treatment than cutaneous lesions as noted similar to that of a prospective study. ${ }^{13}$ Delayed healing might be attributed to poor oral hygiene, trauma of mastication and coexistence of oral candidiasis led to delay in healing. Adequate measures were done to improve oral hygiene along with institution of antifungals, antivirals and antiseptic mouth washes.

Pemphigus foliaceus and bullous pemphigoid had a good response to treatment than pemphigus vulgaris which may be related to the course and prognosis of the disease.

\section{Limitations of the study}

i. Lack of confirmation with immunofluorescence studies due to resource poor setting

ii. Patients are still being followed up on a long-term basis.

Table 1: Age wise distribution in Study group

\begin{tabular}{|l|c|c|c|c|c|c|c|c|}
\hline \multirow{2}{*}{$\begin{array}{c}\text { Age } \\
\text { in years) }\end{array}$} & \multicolumn{2}{c|}{$\begin{array}{c}\text { Pemphigus } \\
\text { Vulgaris(N=18) }\end{array}$} & \multicolumn{2}{c|}{$\begin{array}{c}\text { Pemphigus } \\
\text { Foliaceus(N=5) }\end{array}$} & \multicolumn{2}{c|}{$\begin{array}{c}\text { Bullous } \\
\text { Pemphigoid(N=3) }\end{array}$} & \multicolumn{2}{c|}{ Total } \\
\cline { 2 - 9 } & $\mathbf{N}$ & $\mathbf{\%}$ & $\mathbf{N}$ & $\mathbf{\%}$ & $\mathbf{N}$ & $\mathbf{\%}$ & $\mathbf{N}$ & $\mathbf{\%}$ \\
\hline $15-40$ & 6 & 33.33 & 2 & 40 & 0 & 0 & 8 & 30.77 \\
\hline $40-60$ & 8 & 44.44 & 3 & 60 & 2 & 66.67 & 13 & 50 \\
\hline$>60$ & 4 & 22.22 & 0 & 0 & 1 & 33.33 & 5 & 19.23 \\
\hline
\end{tabular}


Table 2: Sex wise distribution in study group

\begin{tabular}{|l|c|c|c|c|c|c|c|c|}
\hline \multirow{3}{*}{ Sex } & \multicolumn{2}{c|}{$\begin{array}{c}\text { Pemphigus } \\
\text { Vulgaris(N=18) }\end{array}$} & \multicolumn{2}{c|}{$\begin{array}{c}\text { Pemphigus } \\
\text { Foliaceus(N=5) }\end{array}$} & $\begin{array}{c}\text { Bullous } \\
\text { Pemphigoid(N=3) }\end{array}$ & \multicolumn{2}{c|}{ Total } \\
\cline { 2 - 9 } & $\mathbf{N}$ & \% & $\mathbf{N}$ & \% & N & \% & N & $\%$ \\
\hline Male & 3 & 16.67 & 2 & 40 & 3 & 100 & 8 & 30.77 \\
\hline Female & 15 & 83.33 & 3 & 60 & 0 & & 18 & 69.23 \\
\hline
\end{tabular}

Table 3: Body surface area involvement in study group

\begin{tabular}{|l|c|c|}
\hline $\begin{array}{c}\text { Body surface area } \\
\text { (in percentage) }\end{array}$ & $\begin{array}{c}\text { No of patients } \\
(\mathbf{N = 2 6 )}\end{array}$ & $\begin{array}{c}\text { Percentage } \\
(\boldsymbol{\%})\end{array}$ \\
\hline $10-25$ & 5 & 19.23 \\
\hline $25-50$ & 7 & 26.92 \\
\hline$>50$ & 14 & 53.85 \\
\hline
\end{tabular}

Table 4: Vesicobullous subtypes and pulse regimens

\begin{tabular}{|l|c|c|c|c|c|c|c|c|}
\hline \multirow{3}{*}{ Subtype } & $\begin{array}{c}\text { Pemphigus } \\
\text { vulgaris(N=18) }\end{array}$ & \multicolumn{2}{c|}{$\begin{array}{c}\text { Pemphigus } \\
\text { foliaceus(N=5) }\end{array}$} & $\begin{array}{c}\text { Bullous pemphigoid } \\
\text { (N=3) }\end{array}$ & \multicolumn{2}{|c|}{ Total } \\
\cline { 2 - 9 } & $\mathbf{N}$ & $\%$ & $\mathbf{N}$ & $\%$ & N & \% & N & $\%$ \\
\hline DCP & 15 & 83.33 & 5 & 100 & 2 & 66.67 & 22 & 84.62 \\
\hline DP & 2 & 11.11 & 0 & & 1 & 33.33 & 3 & 11.54 \\
\hline DAP & 1 & 5.56 & 0 & & 0 & 0 & 1 & 3.85 \\
\hline
\end{tabular}

Table 4: Minor side effect profile of various pulse regimens

\begin{tabular}{|l|l|l|}
\hline \multicolumn{1}{|c|}{ Side effects } & $\begin{array}{c}\text { No of } \\
\text { patients(N=26) }\end{array}$ & $\begin{array}{c}\text { Percentage } \\
(\%)\end{array}$ \\
\hline Weakness \& lethargy & 16 & 61.54 \\
\hline Nausea \& vomiting & 9 & 34.62 \\
\hline Headache \& sleep disturbances & 12 & 46.15 \\
\hline Fever with rigor & 6 & 23.08 \\
\hline Palpitation & 8 & 30.77 \\
\hline Hiccup & 6 & 23.08 \\
\hline Weight gain & 10 & 38.46 \\
\hline Infections & 3 & 11.54 \\
\hline Impaired glucose tolerance & 8 & 30.77 \\
\hline Electrolyte abnormalities & 7 & 26.92 \\
\hline Amenorrhoea & 8 & 44.44 \\
\hline Cataract & 1 & 3.85 \\
\hline Hypertension & 4 & 15.38 \\
\hline Hyperpigmentation & 3 & 11.54 \\
\hline
\end{tabular}

Table 5: Major side effect profile of various pulse regimens

\begin{tabular}{|l|c|c|}
\hline \multicolumn{1}{|c|}{ Side effects } & $\begin{array}{c}\text { No of } \\
\text { patients(n=26) }\end{array}$ & $\begin{array}{c}\text { Percentage } \\
(\mathbf{\%})\end{array}$ \\
\hline Macular choroiditis & 1 & 3.85 \\
\hline Avascular necrosis of femur & 1 & 3.85 \\
\hline $\begin{array}{l}\text { Reactivation of pulmonary } \\
\text { tuberculosis }\end{array}$ & 1 & 3.85 \\
\hline
\end{tabular}

\section{Conclusion}

The results of this study emphasize a positive outcome of DCP therapy in autoimmune vesicobullous disorders especially in pemphigus and can be an excellent therapeutic strategy in resource poor settings for pemphigus, provided strict compliance of patients to DCP schedule, for which proper and adequate counselling will be of great value. DAP is of a therapeutic boon for vesicobullous patients with associated infertility and young unmarried individuals. Regular bedside investigations like Tzanck, KOH mount, gram stain might be very useful in establishing the cause for recurrences and to manage promptly, thereby ensuring early recovery and quick healing. 
Funding: No funding sources.

Conflict of interest: None declared.

\section{References}

1. Pasricha JS, Gupta R. Pulse therapy with dexamethasonecyclophosphamide in pemphigus. Indian J Dermatol Venereol Leprol 1984;50:199-203.

2. Korman NJ. New immunomodulating drugs in autoimmune blistering diseases. Dermatol Clin 2001;19:637-48.

3. Pasricha JS, Khaitan BK. Curative treatment of pemphigus. Arch Dermatol 1996;132:1518-19.

4. Micali G, Musumeci ML, Nasca MR. Epidemiological analysis and clinical course of 84 consecutive case of pemphigus in Eastern Sicily. Int J Dermatol 1998;37(3):197-200.

5. Kyriakis K, Tosca A, Lehou J. A five-year retrospective study on pemphigus and pemphigoid. Australas $J$ Dermatol 1989;30:33-6.

6. Coombs JN, Taylor R, Wilcken N, Florica J, BoyagesJ,et al. Hormone replacement therapy and breast cancer risk in California. Breast $J$ 2005;11:410-15.

7. Pasricha JS, Khaitan BK, Raman RS, Chandra M. Dexamethasone -cyclophosphamide in pemphigus. Indian J Dermatol Venereol Leprol 1984;50:199-203.

8. Dogra A, Gupta S, Khurana S. Dexamethasonecyclophosphamide pulse therapy in pemphigus: a fiveyear retrospective study. Int J Dermatol 2003;48:71-4.

9. Mahajan VK, Sharma NL, Sharma RC. Twelve-year clinico-therapeutic experience in pemphigus: a retrospective study of 54 cases. Int J Dermatol 2005;44:821-7.

10. Pasricha JS. Pulse therapy as a cure for autoimmune diseases. Indian J Dermatol Venereol Leprol 2003;69:323-8.
11. Ratnam KV, Phay KL, Tan CK. Pemphigus therapy with oral prednisolone regimens. Int J Dermatol 1990;29:36367.

12. Jerome LS, Jeifer ESK, Mathew JS. Cytotoxic and antimetabolite agents in dermatology. In: Freedberg IM, Eisen AZ, Wolff K, Austen KF, Golsmith L, Katz SI, eds. Dermatology in general medicine.5th edition. New York: McGraw-Hill; 1999:2797-810.

13. Zivanovic D, Medenica L, Tanasilovic S, Vesic S, Skiljevic D, Tomovic M et al. DexamethasoneCyclophosphamide pulse therapy in Pemphigus. A review of 72 cases. Am J Clin Dermatol 2010;11(2):123-29.

14. Gokhale NR, Mahajan PM, Sule RR. The use of oral cyclophosphamide with dexamethasone pulse therapy in the treatment of pemphigus vulgaris. J Dermatol Treat 2007;18(5):275-78.

15. Hahn BH. Systemic lupus erythematosus. In: Eugene B, Fauci AS, Kasper DL, Hauser SL, Longo DL, Jameson JL, eds. Harrison's principles of internal medicine.15th edition. New York: McGraw-Hill; 2001:1922-8.

16. Kandan S, Thappa DM. Outcome of dexamethasonecyclophosphamide pulse therapy in pemphigus. A case series. Indian J Dermatol Venereol Leprol 2009;75:373 78.

17. Pasricha JS, Poonam. Current regimen of pulse therapy for pemphigus. Minor modifications, improved results. Indian J Dermatol Venereol Leprol 2008;74:217-

18. Pasricha JS. Pulse therapy as a cure for autoimmune diseases. Indian J Dermatol Venereol Leprol 2003;69:323-28.

How to cite this article: K. Kannambal, S. Prasad, K. Kaviarasan, B. Poorana, D. Ranjani. A study on the outcome of pulse therapy in vesicobullous disorders at a tertiary care centre in Chidambaram, Tamilnadu. Indian J Clin Exp Dermatol 2018;4(4):292-96. 